Roberto Guidotti

Miguel Fernández Ruiz

Aurelio Muttoni

\title{
Durchstanzen von Flachdecken bei hohen Stützenlasten
}

Sollen Hochbauten eine flexible Raumnutzung erlauben, stellen Systeme aus Stahlbeton-Flachdecken und Stützen eine wirtschaftliche und effiziente Lösung dar. Die Stützen dieser Bauten werden häufig aus hochfestem Beton vorfabriziert und stark bewehrt, um hohe Normalkräfte mit minimalen Querschnitten aufnehmen zu können. Der oft aus normalfestem Beton bestehende Deckenteil, der zwischen den übereinanderstehenden Stützen liegt, wird dabei ganz beträchtlichen Druckbeanspruchungen ausgesetzt. Zudem erfährt die Deckenplatte um die Stütze eine starke Querkraftbeanspruchung, so dass der Durchstanzwiderstand maßgebend werden kann. Der vorliegende Aufsatz untersucht die Interaktion zwischen den vertikalen Druckbeanspruchungen in der Deckenplatte und dem Durchstanzwiderstand mit Hilfe einer Versuchsreihe an sechs Plattenausschnitten $(3,00 \times 3,00 \times 0,25 \mathrm{~m})$ unter verschiedenen Lastkombinationen. Aufgrund der Versuchsresultate wird ein einfaches Bemessungsmodell vorgestellt. Dieses Modell basiert auf dem Ansatz der DIN 1045-1 für den Durchstanzwiderstand von Decken ohne Querbewehrung, die einer horizontalen Druckspannung ausgesetzt sind (z. B. Vorspannung). Die mit dem Modell errechneten Resultate stimmen gut mit jenen der Versuche überein - sowohl beim Widerstand wie bei der beobachteten Bruchart.

\section{Interaction between column loading and punching shear strength in flat slabs without transverse reinforcement} Flat slabs supported by columns are cost efficient solutions for high-rise buildings. To reduce the cross section of the columns, the columns are typically precast with high strength concrete and include large amounts of longitudinal reinforcement. On the contrary, the slab between columns is typically cast-in-place with normal strength concrete. Thus, the slab is subjected in the support region of the columns to large transverse compressive stresses, which may exceed its uniaxial compressive strength. In addition, the flat slab around the column region has to carry large shear stresses, which may potentially lead to punching shear failures. In this paper, the interaction between column loads and shear forces in the support region of a flat slab is investigated by means of an experimental programme consisting of 6 slabs without transverse reinforcement $(3.0 \times 3.0 \times 0.25 \mathrm{~m})$. The flexural reinforcement of the slab was varied as well as the ratio between the column loads and the applied shear forces. On the basis of these results, a theoretical model based on the punching shear approach of DIN 1045-1 is presented. This model accounts for the compressive stresses in the support region due to column loads and for the potential increase of punching shear strength due to it. A very good agreement between the test results and the theoretical model is obtained both in terms of strength and observed failure modes.

\section{Einleitung}

Beim Bau mehrgeschossiger Hochbauten, wie sie Bild 1 zeigt, liegen die Stahlbetondecken oft auf stark bewehrten Stützen aus hochfestem Beton mit kleinen Abmessungen, die sehr hohe Lasten übertragen. Die Stützen werden vor allem deshalb so ausgebildet, um den von ihnen beanspruchten Platz zu reduzieren, was gerade in den unteren

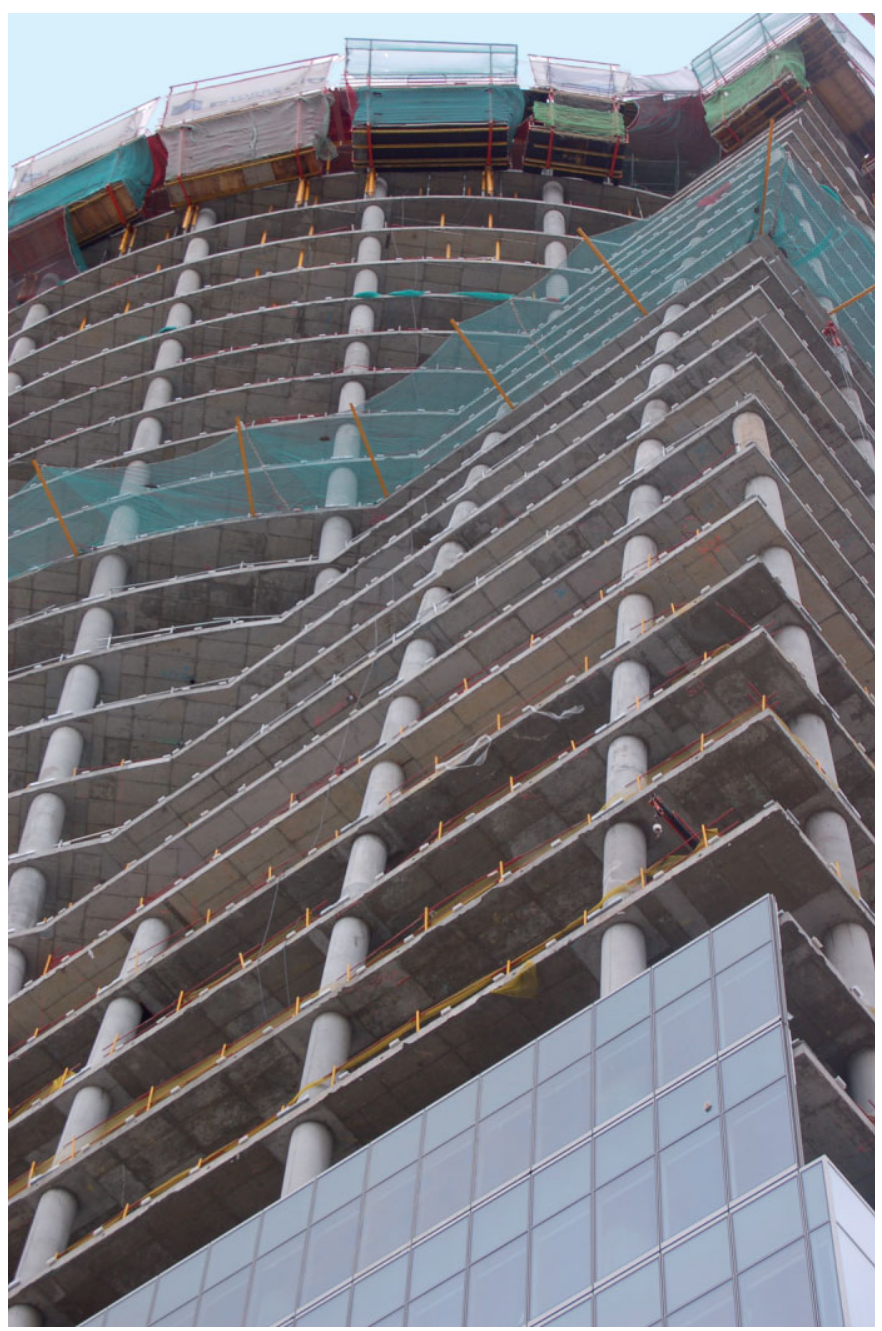

Bild 1. Hochhaus Torre Espacio (Madrid, Spanien), ein Beispiel für Stützen aus hochfestem Beton, die von Decken unterbrochen werden

Fig. 1. Torre Espacio building (Madrid, Spain), an example of high strength concrete columns interrupted by flat slabs 
Geschossen hoher Gebäuden von Bedeutung ist. Aus praktischen Gründen reichen die Stützen zudem oft nur von Decke zu Decke und laufen nicht durch. Daher wird der zwischen den Stützen liegende Bereich der Deckenplatte stark von der Normalkraft der Stützen beansprucht, und die Spannung in der Deckenplatte kann die einachsige Betondruckfestigkeit überschreiten. Außerdem wird die Platte um die Stütze von hohen Querkräften, die von den Deckenlasten herrühren, beansprucht. Diese Querkräfte können zu einem Durchstanzversagen führen und sind oft maßgebend für die Bemessung dieses Bereichs [1]. Damit kann sich im Auflagerbereich der Stützen eine Interaktion von zwei sehr starken Beanspruchungen (Normalkraft in der Stütze und Querkraft in der Deckenplatte) ergeben.

Um diese Problematik zu entschärfen, sind verschiedene konstruktive Details entwickelt worden, die heute in der Praxis ihre Anwendung finden. Bild 2 zeigt die am häufigsten verwendeten konstruktiven Lösungen. Bild 2a stellt die klassische Lösung dar, bei der Stütze und Decke vor Ort betoniert werden. Diese Lösung wird meist bei einer geringen Normalkraft in der Stütze gewählt, die ohne zusätzliche Maßnahmen vom Beton der Decke und der vertikalen Bewehrung aufgenommen werden kann. Bei höheren Normalkräften, die den Widerstand des Betons der Decke und der Vertikalbewehrung überschreiten, kann für die Decke im Stützenbereich ein höherwertiger Beton verwendet (Bild 2b) oder der lokale Widerstand kann mit einer zusätzlichen Umschnürungsbewehrung erhöht werden, siehe Bild 2c.

Bei vorfabrizierten Stützen, die an ihren Enden Stahlplatten aufweisen (Bild 2d-g), können ähnliche Lö- sungen ins Auge gefasst werden. Bei mäßigen Druckkräften kann die Stützenbewehrung bis zur Oberkante der Decke verlängert und mit einer dort montierten Stahlplatte verschweißt werden (Bild 2d). Bei höheren Druckkräften sind konstruktive Lösungen mit Stahlelementen, die die Kräfte durch die Platte leiten (Bild 2e), oder eine Verlängerung der Stuitze denkbar (Bild 2f). In diesem letzteren Fall kann die Oberfläche der Stütze in der Kontaktzone mit der Decke aufgeraut werden, um ein Abtragen der Querkraft zu ermöglichen [2] bis [4].

Die simpelste Lösung bleibt aber jene, bei der die Stuitze einfach auf die Decke gestellt wird (Bild 2g). In diesem Fall werden die Stützenlasten vom Beton der Decke abgetragen, wobei die horizontale Biegebewehrung und die Biegedruckzone der Platte einen Umschnürungseffekt gewährleisten können. Die Hauptfunktion der Biegebewehrung besteht indes weiterhin darin, die Plattenbiegung aufzunehmen und gleichzeitig den Durchstanzwiderstand zu gewährleisten [1], [4]. Damit lässt sich im Auflagerbereich eine Interaktion dreier Phänomene (vertikaler Druckwiderstand, Biegewiderstand der Decke und Durchstanzwiderstand) beobachten. Diese Interaktion und ihre Auswirkungen beim Entwurf und der Bemessung von Stützen-Deckenknoten sind bislang nur wenig bekannt und werden im Folgenden näher beschrieben.

Mehrere Forscher [5], [6] haben den Druckwiderstand von Stützen-Deckenknoten untersucht, ohne dabei die Deckenbiegung zu berücksichtigen (Bild 3a). Andere [7] bis [9] wiederum haben die Abnahme des Druckwiderstands der Stütze im Fußbereich erforscht, der sich aus der biegebedingten horizontalen Dehnung der Platten-
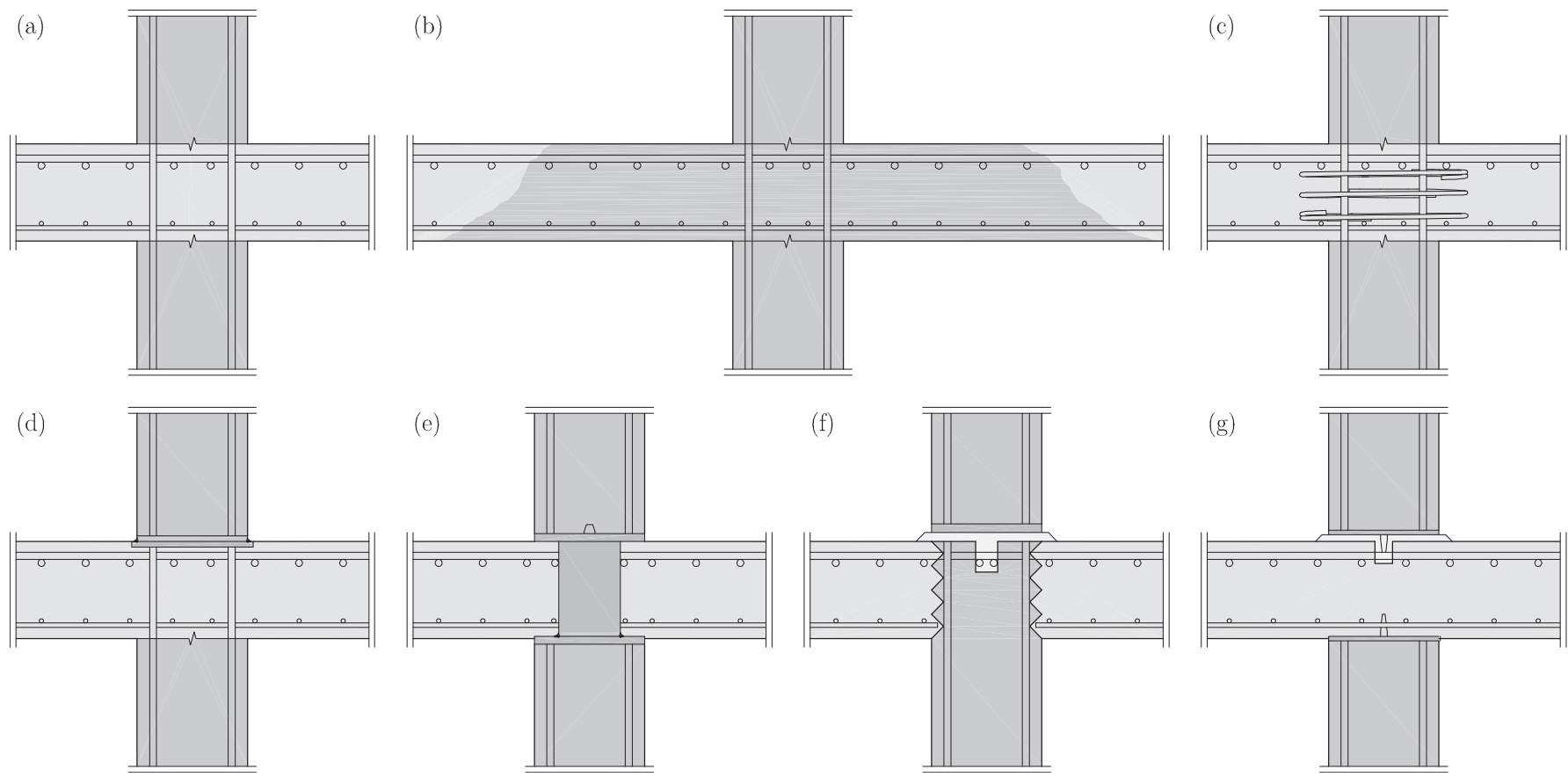

Bild 2. Varianten für die Ausbildung von Stützen-Deckenknoten: (a) Stützen und Decke aus verschiedenen Betonfestigkeiten vor Ort betoniert; (b) Wechsel der Betonfestigkeit in der Decke in Knotennähe; (c) Decke mit eingelegter Umschnürbewehrung; (d) vorgefertigte Stützen und vor Ort betonierte Decke; (e) Verbindung der beiden Stützen mit Stahlelementen; (f) Durchdringung der Decke mit der Stütze [3], [4]; und (g) oben und unten mit Stahlplatten versehene Stützen ohne besondere Maßnahme in der Decke zur Durchleitung der Normalkraft

Fig. 2. Possibilities for column-slab joints: (a) column and slab cast-in-place with different concrete strengths; (b) casting of flat slab near the support region with high-strength concrete; (c) confining reinforcement in flat slab; (d) precast column; (e) joint with steel element used to carry the compressive forces of the column; (f) joint element with shear keys [3], [4]; and (g) columns with steel plates at the top and bottom ends 


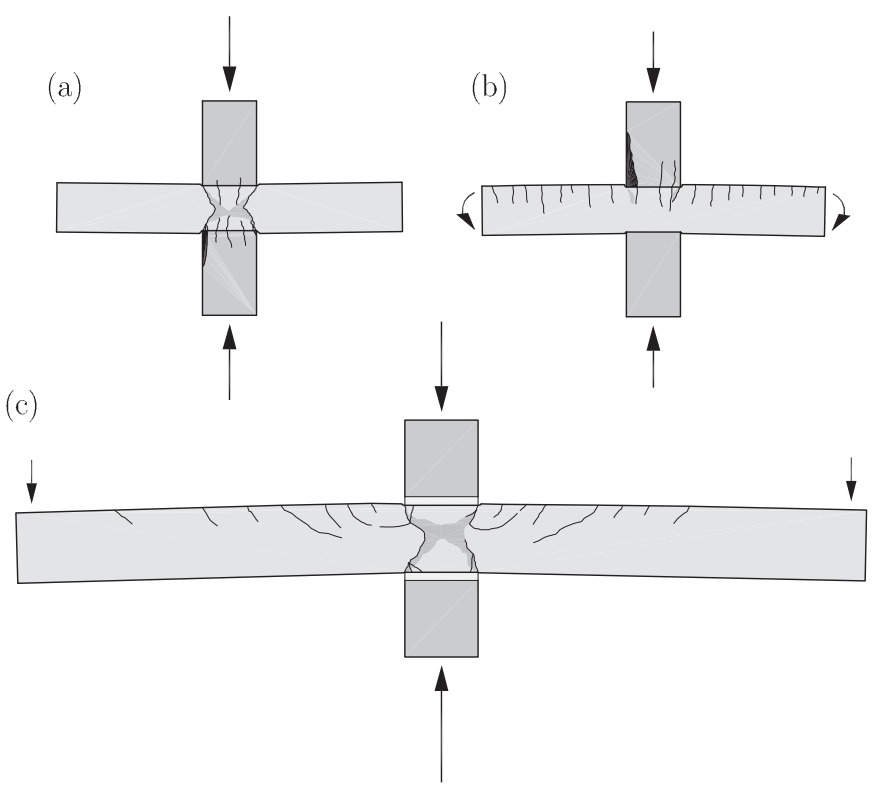

Bild 3. Möglichkeiten der Versuchsdurchführung an Stützen-Deckenknoten: (a) Lastaufbringung nur auf Stützen; (b) Belastung der Stützen mit Aufbringen eines Moments an der Platte; und (c) Belastung der Stützen und der Plattenränder

Fig. 3. Tests on slab-column joints: (a) column loading; (b) column loading and bending moment applied to slab; and (c) column loading and shear forces applied to the slab

oberseite ergibt (Bild 3b). Diese Studien lassen sich jedoch nicht vollständig auf die Situation bei Flachdecken übertragen, wo der Bereich um die Stütze herum auch stark von Querkräften beansprucht wird. Um für diese Fälle realistischere Bedingungen zu reproduzieren, wurden an der ETH Lausanne Versuche an Plattenausschnitten mit herkömmlichen Abmessungen unternommen. Die Plattenelemente werden von den Stützennormalkräften und von an den Plattenrändern wirkenden Kräften beansprucht, welche die für den Auflagerbereich von Flachdecken typischen Biegemomente und Querkräfte erzeugen (Bild 3c). Dabei wurde die in Bild 2g beschriebene konstruktive Lösung untersucht (mit Stahlplatten an beiden Enden der vorfabrizierten Stützen, ohne die Decke durchdringende Vertikalbewehrung und ohne Querbewehrung als Querkraftverstärkung).

Der vorliegende Artikel beschreibt diese Versuchsresultate und schlägt eine auf der DIN 1045-1 beruhende Bemessungsmethode vor, um den Durchstanznachweis unter Berücksichtigung des Einflusses der Normalkraft in der Stütze zu erbringen.

\section{Versuche 2.1 Prüfkörper}

Eine Reihe von sechs Plattenelementen, die den Bereich der Flachdecke auf einer Innenstütze abbilden, wurde an der Eidgenössischen Technischen Hochschule Lausanne untersucht (Bild 4). Alle untersuchten Elemente waren quadratisch $(3,00 \times 3,00 \mathrm{~m})$, wiesen eine Dicke von $0,25 \mathrm{~m}$ und eine nominelle statische Höhe von 0,21 m auf. Die Abmessungen der quadratischen Stahlplatten an den Stützenenden betrugen $0,26 \times 0,26 \mathrm{~m}$.
Drei Prüfkörper waren mit einem Biegebewehrungsgehalt von $\rho_{\text {nom }}=0,8 \%$ bewehrt, während die anderen einen solchen von $\rho_{\text {nom }}=1,6 \%$ aufwiesen. Für beide Bewehrungsgehalte wurde je ein Versuch ohne Normalkraft (Referenzversuch), ein zweiter mit einer mittleren Normalkraft $\mathrm{N}$ in der Stütze und ein dritter mit einer hohen Normalkraft N durchgeführt. Die Platten verfügten weder über eine Durchstanzbewehrung noch eine Bewehrung zum Abtragen der Vertikalkraft aus den Stützen (Bild 4a). Tabelle 1 enthält für jeden Prüfkörper den effektiven Biegebewehrungsgehalt $\rho$, die effektive mittlere statische Höhe d (gemessen am aufgeschnittenen Element) und die Normalkraft $\mathrm{N}_{\mathrm{u} \text {,test }}$ beim Bruch.

\subsection{Versuchsdurchführung}

Die Normalkraft $\mathrm{N}$ und die Querkraft V wurden gleichzeitig aufgebracht und in der Regel proportional bis zum Bruch erhöht. Bei den einer hohen Normalkraft $\mathrm{N}$ unterzogenen Prüfkörpern wurde nach Erreichen einer Normalkraft N von ungefähr 7,3 MN nur noch die Querkraft V erhöht. Vier hydraulische Pressen erzeugten die Querkraft (V) und übertrugen sie über ein System von Stahlstäben und Verteilträgern auf acht am Rand des Plattenelements angeordnete Stahlplatten (Bild 4b, siehe auch [10]). Die Normalkraft $\mathbf{N}$ wurde von vier hydraulischen Pressen mit einer Kapazität von je 2 MN erzeugt. Vier Rückhaltestangen mit $\varnothing 76 \mathrm{~mm}$ durchdrangen das Plattenelement in genügendem Abstand von der Stütze, um die Bruchzone nicht zu beeinflussen. Die Kraft wurde von zwei stählernen Verteilträgern auf zwei Stahlplatten übertragen, die die Stützenenden darstellten (Bild 4c).

\subsection{Baustoffeigenschaften}

Die Prüfkörper bestanden aus einem normalfesten Beton. Bei den Versuchen mit $\rho_{\text {nom }}=0,8 \%$ variierte die Zylinderdruckfestigkeit zwischen 31,5 und $34,5 \mathrm{~N} / \mathrm{mm}^{2}$, während sie bei den Versuchen mit $\rho_{\text {nom }}=1,6 \%$ zwischen 47,3 und $51,7 \mathrm{~N} / \mathrm{mm}^{2}$ lag (siehe Details in Tabelle 1). Das Größtkorn der Gesteinskörnung maß bei allen Elementen $16 \mathrm{~mm}$. Für die Biegebewehrung wurden naturharte Stähle (mit Fließplateau) verwendet. Die Fließgrenze lag zwischen 538 und $551 \mathrm{~N} / \mathrm{mm}^{2}$ (siehe Tabelle 1).

\subsection{Versuchsresultate}

Die Diagramme (Bild 5) zeigen die Lastverformungskurven $(\mathrm{V}-\psi)$ der Platten sowie die sich daraus ergebenden Lastpfade $(\mathrm{N}-\mathrm{V})$. Die dargestellte Verformung entspricht der Rotation $\psi$ der Platte außerhalb des Stützenbereichs (siehe Darstellung in Bild 5a). Gemäß [1] gilt die Plattenrotation $\psi$ als repräsentative Größe für die Verformung im Bruchzustand, weil sich die Platte im Stützenbereich etwa kegelförmig verbiegt. Bei fünf der sechs Versuchen erfolgte der Bruch aufgrund des Durchstanzens der Stütze, während sich bei einem Versuch ein Biegemechanismus einstellte. Die Resultate zeigen interessanterweise einen mit der Normalkraft zunehmenden Durchstanzwiderstand. Diese Erscheinung wird begleitet von einer Zunahme der Biegeverformungen der Platte (Rotation $\psi$ ). Diese Zunahmen von Widerstand und Verformung werden relevant, 
(a)

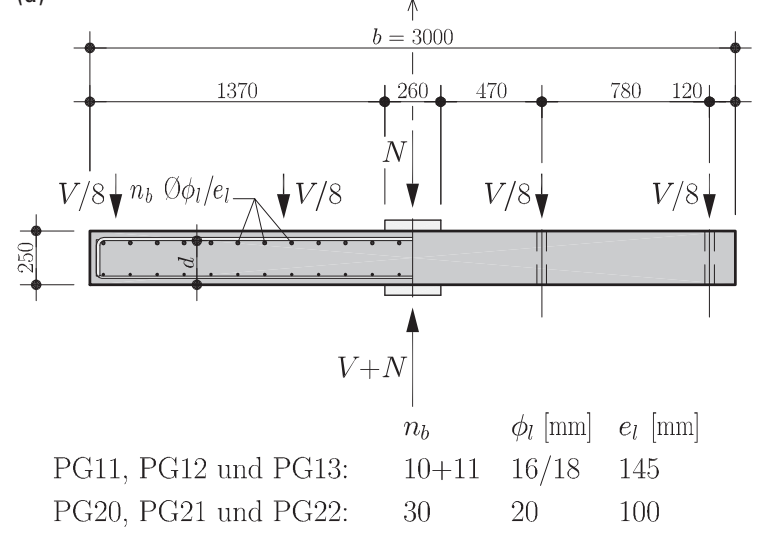

(b)

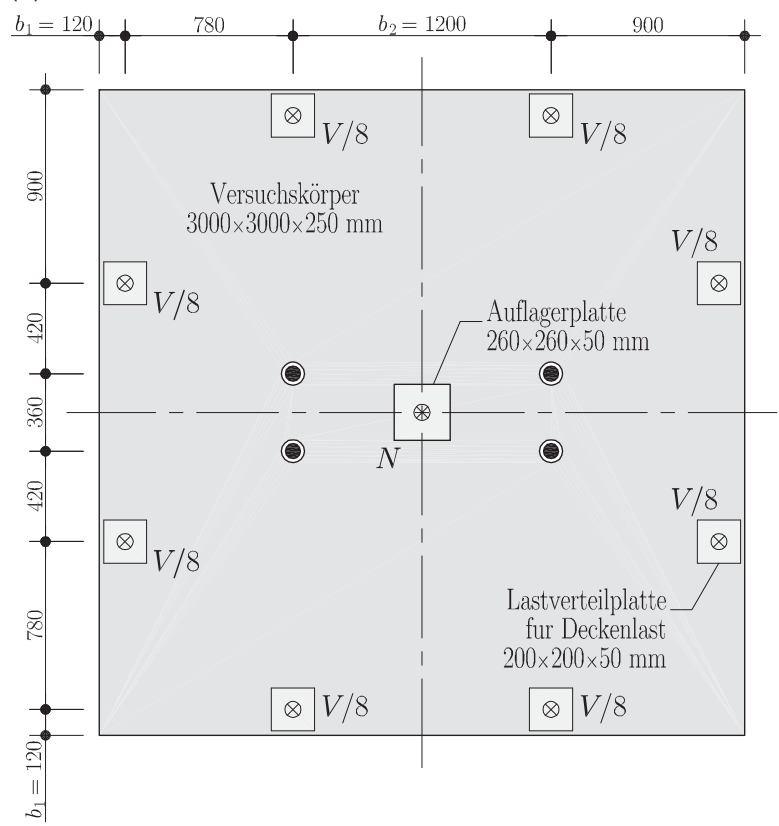

(c)
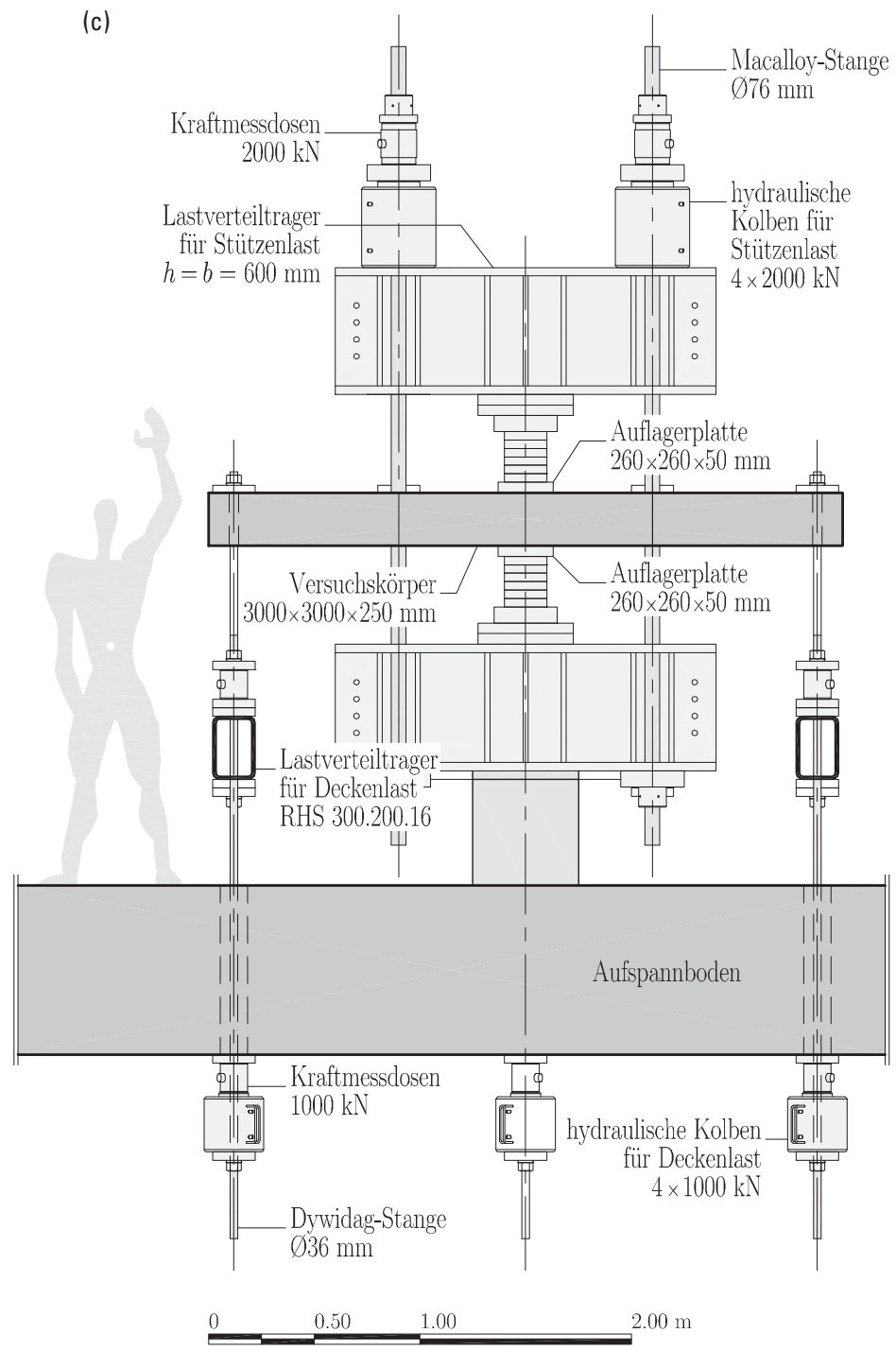

Bild 4. Versuchskörper und Versuchsaufbau: (a) Querschnitt der Platte; (b) Grundriss des Plattenelements mit den Lasteinleitungsplatten; und (c) Versuchsaufbau

Fig. 4. Test specimen and test setup: (a) cross section of the specimen; (b) plan view of the specimen and loading plates; and (c) view of test setup

sobald die aus der Normalkraft resultierende Vertikalspannung

$\sigma_{\mathrm{c}, \mathrm{v}}=\mathrm{N} / \mathrm{A}_{\mathrm{c}}$

$\left(\mathrm{A}_{\mathrm{c}}\right.$ ist die Stützenfläche) die Zylinderdruckfestigkeit $\mathrm{f}_{\mathrm{c}, \mathrm{zyl}}$ des Betons in der Decke deutlich überschreitet.

Beim Versuch PG21 ( $\rho=1,6 \%$ mit mäßiger Normalkraft), bei dem die Vertikalspannung $\sigma_{c, v}$ beim Bruch nur $1,32 f_{c, z y l}$ betrug, war der Durchstanzwiderstand praktisch gleich groß wie beim Referenzversuch ohne Normalkraft. Beim Versuch PG22 ( $\rho=1,6 \%$ mit hoher Normalkraft), bei dem die Vertikalspannung $\sigma_{\mathrm{c}, \mathrm{V}}$ mehr als das Doppelte der Druckfestigkeit $\left(\sigma_{\mathrm{c}, \mathrm{v}}=2,22 \mathrm{f}_{\mathrm{c}, \mathrm{zyl}}\right)$ betrug, nahm der Durchstanzwiderstand hingegen beträchtlich zu (um 31\% gegenüber der Platte ohne Normalkraft).

Beim Versuch PG12 ( $\rho=0,8 \%$ mit mäßiger Normalkraft, $\left.\sigma_{\mathrm{c}, \mathrm{v}}=1,66 \mathrm{f}_{\mathrm{c}, \mathrm{zyl}}\right)$ nahm der Durchstanzwiderstand gegenüber dem Referenzelement um 25\% zu.

Beim Versuch PG13 ( $\rho=0,8 \%$ mit hoher Normalkraft) erreichte die Vertikalspannung $\sigma_{\mathrm{c}, \mathrm{V}}$ beinahe das
Tabelle 1. Übersicht der Versuchsparameter und Resultate Table 1. Main parameters and results

\begin{tabular}{l|c|c|c|c|c|c|c|c}
\hline Decke & $\begin{array}{c}\rho \\
{[\%]}\end{array}$ & $\begin{array}{c}\mathrm{d} \\
{[\mathrm{mm}]}\end{array}$ & $\begin{array}{c}\mathrm{f}_{\mathrm{c}, \mathrm{zyl}} \\
{\left[\mathrm{N} / \mathrm{mm}^{2}\right]}\end{array}$ & $\begin{array}{c}\mathrm{f}_{\mathrm{y}} \\
{\left[\mathrm{N} / \mathrm{mm}^{2}\right]}\end{array}$ & $\begin{array}{c}\mathrm{N}_{\mathrm{u}, \text { test }} \\
{[\mathrm{kN}]}\end{array}$ & $\begin{array}{c}\mathrm{N}_{\mathrm{u}, \text { test }} / \\
\left(\mathrm{A}_{\mathrm{c}} \mathrm{f}_{\mathrm{c}, \mathrm{zyl}}\right)\end{array}$ & $\begin{array}{c}\mathrm{V}_{\mathrm{u}, \text { test }} \\
{[\mathrm{kN}]}\end{array}$ & $\begin{array}{l}\psi_{\mathrm{u}, \text { test }} \\
{[\%]}\end{array}$ \\
\hline PG11 & 0,77 & 208 & 31,5 & 538 & 0 & 0 & 763 & 10,3 \\
\hline PG12 & 0,81 & 198 & 34,5 & 538 & 3868 & 1,66 & 957 & 21,0 \\
\hline PG13 & 0,82 & 195 & 33,9 & 538 & 6611 & 2,88 & 1027 & 29,9 \\
\hline PG20 & 1,56 & 201 & 51,7 & 551 & 0 & 0 & 1094 & 9,2 \\
\hline PG21 & 1,61 & 195 & 47,3 & 551 & 4227 & 1,32 & 1105 & 10,3 \\
\hline PG22 & 1,57 & 200 & 48,4 & 551 & 7275 & 2,22 & 1428 & 17,2 \\
\hline
\end{tabular}

Dreifache der Betondruckfestigkeit $\left(\sigma_{\mathrm{c}, \mathrm{v}}=2,88 \mathrm{f}_{\mathrm{c}, \mathrm{zyl}}\right)$ und der Durchstanzwiderstand erhöhte sich dermaßen, dass sich ein Biegemechanismus einstellte. Der Versuch musste nach sehr großen Verformungen und bei einer Querkraft, die den Durchstanzwiderstand des Referenzelements um $31 \%$ überschritt, abgebrochen werden. 

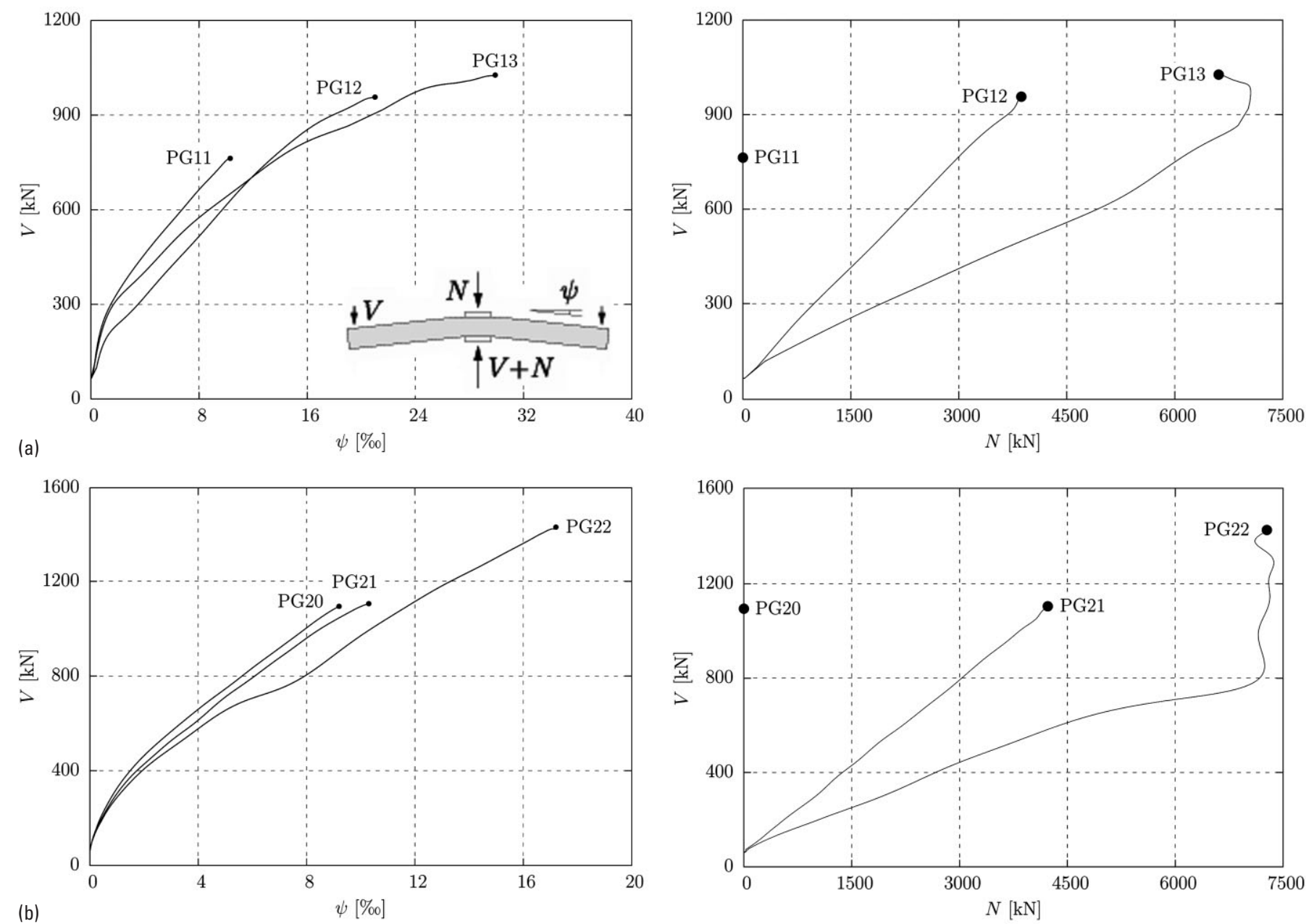

Bild 5. Versuchsresultate: Lastverformungskurven $(V-\psi)$ und Normalkraftbelastung $(V-N)$ für: (a) Platten mit $\rho_{\text {nom }}=0,8 \%$; und (b) Platten mit $\rho_{\text {nom }}=1,6 \%$

Fig. 5. Test results: Load-rotation curve $(V-\psi)$ and axial load curve $(V-N)$ for: (a) slabs with $\rho_{\text {nom }}=0.8 \%$; and $(b)$ slabs with $\rho_{\text {nom }}=1.6 \%$

\subsection{Fazit aus den Versuchen und Interpretation}

In erster Linie zeigen die Versuche, dass die in der Platte im Auflagerbereich wirkende Vertikalspannung, aufgrund des Einschnürungseffekts durch die Platte, die Betondruckfestigkeit bei weitem übersteigen kann. Dazu muss keine besondere Maßnahme ergriffen werden. Zudem übt die von der Plattenbiegung ausgelöste Querdehnung sogar bei stark plastisch gewordener Biegebewehrung keinen negativen Einfluss auf die Betonfestigkeit der Platte aus.

Überschreitet die Vertikalspannung die Betonfestigkeit, müssen sich Radialspannungen entwickeln, um einen Betonbruch zu verhindern. Diese radialen Druckspannungen üben eine negative Wirkung auf die Biegung aus, weil sie die Biegebewehrung zusätzlich beanspruchen und die Biegeverformungen erhöhen (Rotation $\psi$ ). Gleichzeitig wirken sich diese Radialspannungen, ähnlich wie bei vorgespannten Decken, auf den Durchstanzwiderstand positiv aus.

\section{Berechnungsmodell}

\subsection{Einfluss der Normalkraft auf den Durchstanzwiderstand}

Liegen die Vertikalspannungen aus der Normalkraft unter der Zylinderdruckfestigkeit des Betons, ist die Querverfor- mung gering (Poisson'scher Effekt), so dass die positive Auswirkung auf den Durchstanzwiderstand (in die Platte eingebrachte „Vorspannung“) vernachlässigbar ist. Wird hingegen die einaxiale Betondruckfestigkeit überschritten, muss sich eine radiale Betondruckspannung einstellen (Bild 6). Diese Spannung lässt sich mit einem Mohr-Coulomb'schen Bruchkriterium berechnen. Für einen inneren Reibungswinkel des Betons $\varphi=37^{\circ}$ beträgt sie [11]:

$$
\sigma_{\mathrm{c}, \mathrm{h}}=\frac{1}{4}\left(\sigma_{\mathrm{c}, \mathrm{v}}-\mathrm{f}_{\mathrm{c}}\right) \geq 0
$$

wobei $\mathrm{f}_{\mathrm{c}}$ die Betondruckfestigkeit der Platte ohne Einschnürungseffekt bezeichnet. Kann die Reibung der Stahlplatten auf der Betonoberfläche vernachlässigt werden, lässt sich die einaxiale Betondruckfestigkeit mit $\mathrm{f}_{\mathrm{c}}=\mathrm{f}_{\mathrm{c}, \mathrm{zyl}}$ annehmen, während sie bei vorhandener Reibung erhöht

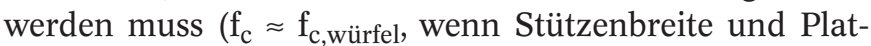
tenstärke ungefähr gleich groß sind). Es ist zu beachten, dass diese Erhöhung für den Durchstanzwiderstand ungünstig ist. Die so berechnete Einschnürungsspannung erzeugt einen radialen Druck, der sich in der Platte und der Biegebewehrung ausbreitet, so dass die Intensität dieser Spannung mit zunehmender Distanz zur Stütze abnimmt. 


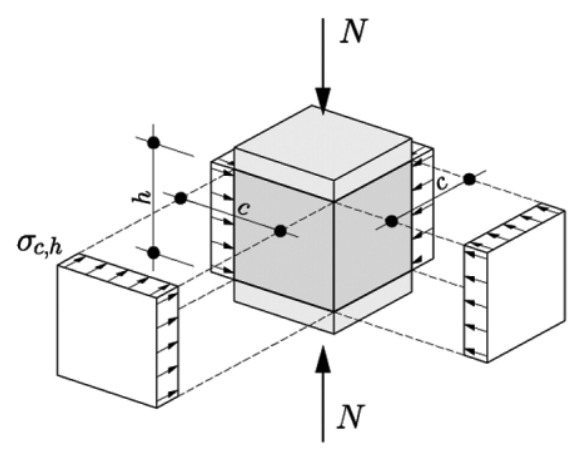

Bild 6. Verteilung der Einschnürungsspannung rund um den Beton zwischen den Lasteinleitungsplatten

Fig. 6. Confining stresses in the support region

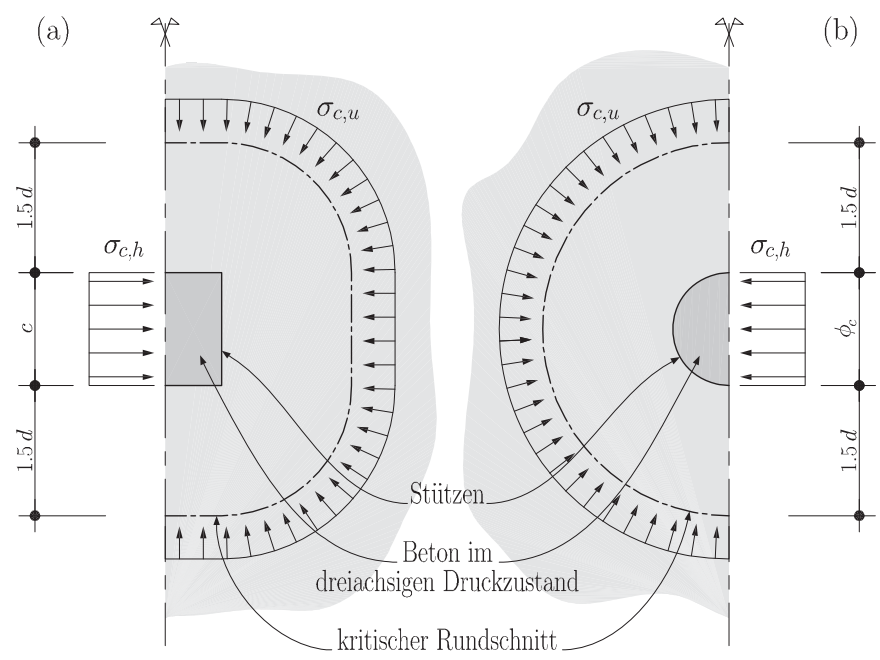

Bild 7. Ausbreitung der Einschnürungsspannung innerhalb des Nachweisschnitts: (a) bei quadratischen Stützen; und (b) bei runden Stützen

Fig. 7. Distribution of confining stresses around the control perimeter: (a) for a square support region; and (b) for a circular support region

Auf dem Nachweisschnitt für das Durchstanzen (im Abstand von 1,5d vom Rand der Auflagerfläche gemäß DIN 1045-1 [12]) beträgt die radiale Druckspannung $\sigma_{\mathrm{c}, \mathrm{u}}$ (Bild 7) :

$\sigma_{\mathrm{c}, \mathrm{u}}=\frac{\sigma_{\mathrm{c}, \mathrm{h}}}{1+3 \cdot \mathrm{d} / \mathrm{b}_{\mathrm{c}}}$

wobei $b_{c}$ die Stützenabmessung bezeichnet (c für quadratische und $\phi_{c}$ für runde Stützen). Gleichung (3) geht von der Annahme aus, dass sich der Radialdruck nur außerhalb des Nachweisschnitts auf die Biegebewehrung überträgt.

Die DIN 1045-1 [12] erlaubt das Berechnen des Durchstanzwiderstands als Funktion dieser Druckspannung $\sigma_{\mathrm{c}, \mathrm{d}}$ :

$\mathrm{V}_{\mathrm{Rd}, \mathrm{ct}}=\mathrm{u} \cdot \mathrm{d} \cdot\left[\frac{0.21}{\gamma_{\mathrm{c}}} \cdot \eta_{1} \cdot \kappa \cdot\left(100 \cdot \rho \cdot \mathrm{f}_{\mathrm{ck}}\right)^{1 / 3}+0.12 \cdot \sigma_{\mathrm{c}, \mathrm{d}}\right]$

wobei u die Länge des Nachweisschnitts, $\kappa$ der Maßstabfaktor, $\mathrm{f}_{\mathrm{ck}}$ die charakteristische Zylinderdruckfestigkeit

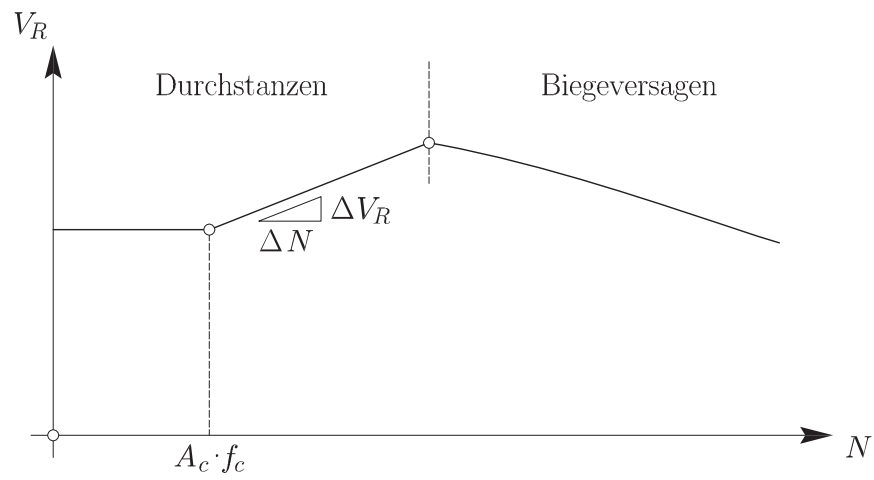

Bild 8. Diagramm der Interaktion $V_{R^{-}} N$

Fig. 8. $V_{R^{-}} N$ interaction diagram

des Betons und $\eta_{1}=1$ für Normalbeton ist. Um diese Formel mit den Versuchen zu vergleichen, kann man $\gamma_{\mathrm{c}}=1$ annehmen, von durchschnittlichen Materialfestigkeiten ausgehen und annehmen, dass die Radialspannung dem berechneten Wert aus den Gln. (2) und (3) entspricht:

$V_{R}=u \cdot d \cdot\left[0.21 \cdot \kappa \cdot\left(100 \cdot \rho \cdot f_{c, z y l}\right)^{1 / 3}+0.12 \cdot \sigma_{c, u}\right]$

Mit diesem Ansatz wird die Zunahme des Durchstanzwiderstands zu einer linearen Funktion der Radialspannung und damit auch der Normalkraft N (Bild 8). Die Neigung dieser Geraden hängt nur vom inneren Reibungswinkel des Betons und der Knotengeometrie ab. Für $\varphi=37^{\circ}$ und eine quadratische Stütze $(c \times c)$ beträgt die Neigung:

$\frac{\Delta \mathrm{V}_{\mathrm{R}}}{\Delta \mathrm{N}}=0.03 \cdot \frac{4+3 \cdot \pi \cdot \mathrm{d} / \mathrm{c}}{3+\mathrm{c} / \mathrm{d}}$

und für runde Stützen mit dem Durchmesser $\phi_{\mathrm{c}}$ :

$\frac{\Delta \mathrm{V}_{\mathrm{R}}}{\Delta \mathrm{N}}=0.12 \cdot \frac{\mathrm{d}}{\phi_{\mathrm{c}}}$

\subsection{Einfluss der Normalkraft auf den Biegewiderstand}

Die radiale Einschnürungsspannung ist im Gleichgewicht mit einer Zunahme der Zugkraft in der Biegebewehrung und einer Verringerung der radialen Kraft in der Biegedruckzone der Platte. Daher bewirkt die Normalkraft eine Zunahme der Biegeverformungen (Zunahme der Rotationen $\psi$ in Bild 5). Im Bruchzustand kann die Normalkraft $\mathrm{N}$ zu einer Verringerung der Biegetraglast führen, wie in Bild 8 gezeigt. Dies lässt sich mit einem Mechanismus nach der Plastizitätstheorie [13] berechnen. Ein solcher Mechanismus, der mit einem einzigen plastischen Gelenk das ganze Plattenelement durchzieht und am Stützenrand liegt, ergibt für die betrachtete Geometrie die folgende Biegetraglast:

$\mathrm{V}_{\text {biegung }}=\frac{8 \cdot \mathrm{b}}{\mathrm{b}+\mathrm{b}_{2}-2\left(\mathrm{~b}_{1}+\mathrm{c}\right)}\left[\begin{array}{l}\mathrm{f}_{\mathrm{y}} \cdot \rho \cdot \mathrm{d} \cdot\left(\mathrm{d}-\frac{\mathrm{h}}{2}\right)+\mathrm{f}_{\mathrm{c}, \mathrm{zyl}} \cdot \\ \frac{(\mathrm{h}-\mathrm{x}) \cdot \mathrm{x}}{2}-\sigma_{\mathrm{c}, \mathrm{h}} \frac{(\mathrm{h}-\mathrm{x}) \cdot \mathrm{c} \cdot \mathrm{x}}{2 \mathrm{~b}}\end{array}\right]$ (8)

wobei $b, b_{1}$ und $b_{2}$ den in Bild 4 angegebenen Abmessungen entsprechen, $\mathrm{h}$ die Plattendicke bezeichnet, $\mathrm{c}$ die Brei- 


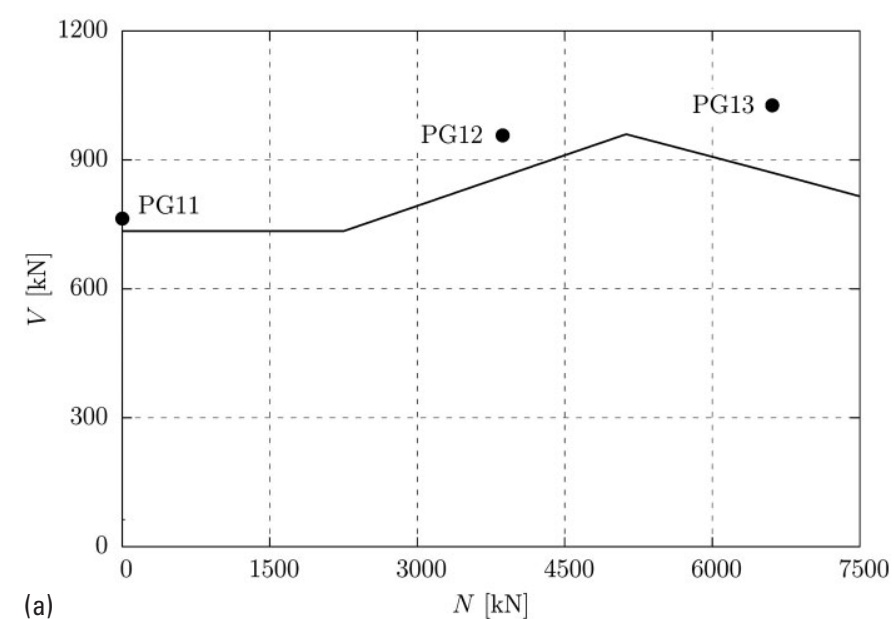

(a)

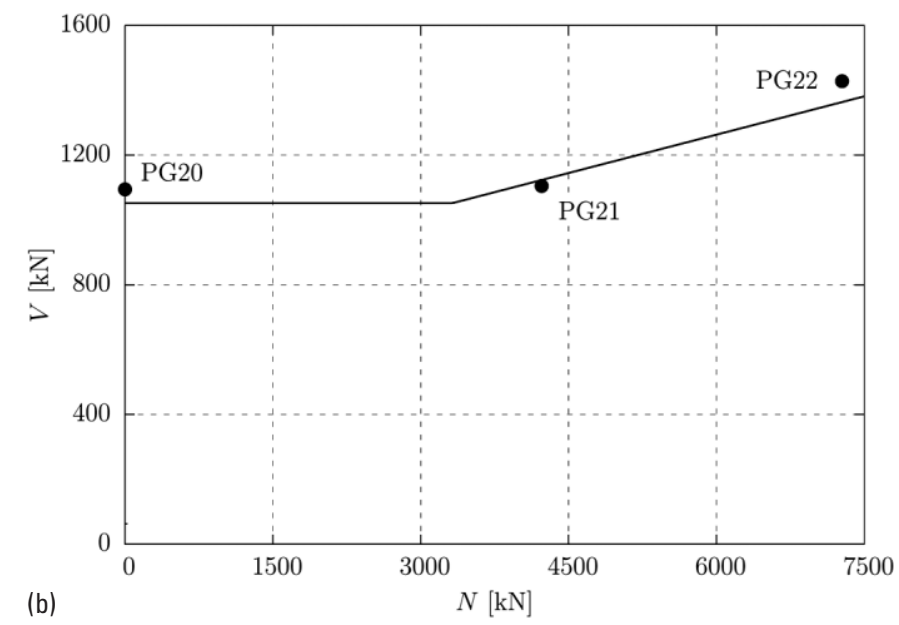

Bild 9. Vergleich der Versuchsresultate mit dem vorgeschlagenen Berechnungsmodell: (a) $\rho=0,80 \%, d=200 \mathrm{~mm}$, $f_{c}=f_{c, z y l}=33,3 \mathrm{~N} / \mathrm{mm}^{2}$; (b) $\rho=1,56 \%, d=201 \mathrm{~mm}$, $f_{c}=f_{c, z y l}=49,4 \mathrm{~N} / \mathrm{mm}^{2}$

Fig. 9. Comparison of test results with the design model:

(a) $\rho=0,80 \%, d=200 \mathrm{~mm}, f_{c}=f_{c, z y l}=33,3 \mathrm{~N} / \mathrm{mm}^{2}$;

(b) $\rho=1,56 \%, d=201 \mathrm{~mm}, f_{c}=f_{c, z y l}=49,4 \mathrm{~N} / \mathrm{mm}^{2}$

te der quadratischen Stütze und $\mathrm{x}$ die Höhe der Druckzone angibt:

$x=\left(f_{y} \cdot \rho \cdot d-\sigma_{c, h} \cdot h \cdot c / b\right) /\left(f_{c, z y l}-\sigma_{c, h} \cdot c / b\right)$

Theoretisch wären für Platten mit sehr starker Biegebewehrung und sehr hohen Normalkräften $\mathrm{N}$ andere Bruchmechanismen, wie ein Betonbruch in radialer Richtung oder das Fließen der unteren Plattenbewehrung, denkbar. Für übliche Geometrien und Bewehrungen sind diese Bruchformen jedoch nicht maßgebend.

\subsection{Vergleich mit den Versuchen}

Bild 9 und Tabelle 2 zeigen einen Vergleich der vorgestellten Versuchsresultate mit den Durchstanzwiderständen und Biegetraglasten, wie sie mit den Gln. (5) und (8) beschrieben sind. Die Ergebnisse zeigen eine ziemlich gute Übereinstimmung. Das mittlere Verhältnis zwischen gemessenem und berechnetem Widerstand liegt bei 1,08 und der Variationskoeffizient für die sechs Versuche beträgt $8 \%$. Die Zuverlässigkeit der vorgeschlagenen Model-
Tabelle 2. Zusammenstellung der gemessenen ( $\left.V_{u, t e s t}\right)$ und berechneten Widerstände $\left(V_{u, c a l c}\right.$, bestimmt aus der beim Bruch gemessenen Normalkraft)

Table 2. Comparison between measured $\left(V_{u, t e s t}\right)$ and calculated $\left(V_{u, \text { calc }}\right)$ shear at failure

\begin{tabular}{l|c|c|c|c}
\hline Decke & Bruchart & $\mathrm{V}_{\mathrm{u}, \text { calc }}[\mathrm{kN}]$ & $\mathrm{V}_{\mathrm{u}, \text { test }}[\mathrm{kN}]$ & $\mathrm{V}_{\mathrm{u}, \text { test }} / \mathrm{V}_{\text {calc }}$ \\
\hline PG11 & Durchstanzen & 752 & 763 & 1,02 \\
\hline PG12 & Durchstanzen & 853 & 957 & 1,12 \\
\hline PG13 & Biegung & 843 & 1027 & 1,22 \\
\hline PG20 & Durchstanzen & 1070 & 1094 & 1,02 \\
\hline PG21 & Durchstanzen & 1078 & 1105 & 1,02 \\
\hline PG22 & Durchstanzen & 1355 & 1428 & 1,05 \\
\hline \multicolumn{5}{c}{ Mittelwert: } \\
\hline \multicolumn{5}{c}{ Variationskoeffizient: } \\
\hline
\end{tabular}

le ist damit vergleichbar mit jener der Modelle für das Durchstanzen von Platten ohne Normalkraft in der oberen Stütze [1]. Auch die Brucharten sowie die Zunahme des Durchstanzwiderstands unter Normalkraft werden korrekt erfasst.

\section{Schlussbemerkungen}

Dieser Beitrag untersucht die Wirkung der Normalkraft in übereinanderstehenden Stützen eines Hochbaus auf den Durchstanzwiderstand und die Biegetraglast einer Platte, die in vertikaler Richtung vollkommen unbewehrt ist. Die Hauptfolgerungen sind:

1. Der Beton der Platte im Stützenbereich kann dank dem Umschnürungseffekt der Platte Druckspannungen widerstehen, die weit über seiner einaxialen Druckfestigkeit liegen.

2. Die durch die Biegung ausgelöste Dehnung der oberen Plattenbewehrung bewirkt auch bei sehr großen Verformungen im plastischen Bereich keine Abnahme der Betondruckfestigkeit in der Platte.

3. Überschreitet die Vertikalspannung die Betondruckfestigkeit der Platte, entwickeln sich horizontale $\mathrm{zu}$ sätzliche Druckspannungen im Auflagerbereich, die sich radial ausbreiten.

4. Diese, von der Normalkraft im Beton ausgelösten, radialen Druckspannungen erhöhen den Durchstanzwiderstand der Platte. Es handelt sich dabei um einen ähnlichen Effekt, wie wenn die Platte vorgespannt wäre.

5. Die Zunahme des Durchstanzwiderstands infolge der Stützennormalkraft lässt sich mit der in der DIN 10451 vorgeschlagenen Gleichung ermitteln.

6. Die Zunahme des Durchstanzwiderstands wird auch von einer merkbaren Zunahme der Verformungsfähigkeit der Platte im Bruchzustand begleitet.

7. Die radialen Druckspannungen im Beton werden durch eine Zunahme der Zugkraft in der Biegebewehrung ausgeglichen, was zu höheren Biegeverformungen und einer Abminderung der Biegetraglast der Platte führt. 


\section{Literatur}

[1] Muttoni A.: Punching shear strength of reinforced concrete slabs without transverse reinforcement, American Concrete Institute, Structural Journal, V. 105, N 4, S. 440-450, 2008.

[2] Mühlbauer S. und Stenzel G.: Kompaktstützen aus hochfestem Beton, Beton- und Stahlbetonbau 98 (2003), Heft 11, S. 678-686, November, 2003.

[3] Rinnhofer G., Burtscher S. L. und Kollegger J.: StützenDeckenknoten für Schleuderbetonstützen, Beton- und Stahlbetonbau 103 (2008), Heft 9, S. 609-616, September, 2008.

[4] Rinnhofer G., Burtscher S. L. und Kollegger J.: Berechnung von Stützen-Deckenknoten für Schleuderbetonstützen, Beton- und Stahlbetonbau 104 (2009), Heft 9, S. 599-608, September, 2009.

[5] Bianchini A. C., Woods R. E. and Kesler C. E.: Effect of Floor Concrete Strength on Column Strength, Journal of the American Concrete Institute, ACI, S. 1149-1169, USA, Mai, 1960.

[6] McHarg P. J., Cook W. D., Mitchell D. and Yoon Y.-S.: Improved Transmission of High-Strength Concrete Column Loads through Normal Strength Concrete Slabs, ACI Structural Journal, Vol. 97, S. 157-165, USA, Januar-Februar, 2000.

[7] Ospina C. E. and Alexander S. D. B.: Transmission of Interior Concrete Column Loads through Floors, Journal of Structural Engineering, ASCE, Vol. 124, No. 6, S. 602-610, USA, Juni, 1998.

[8] Jungwirth F.: Knotenpunkt: normalfeste Decke - hochfeste Ortbetonstütze, Universität Leipzig, LACER, No. 3, S. 165174, 1998.

[9] Tue N. V., Dietz J. und Shah H. C.: Vorschlag für die Bemessung der Deckenknoten mit Stützen aus hochfestem Beton, Beton- und Stahlbetonbau 100 (2005), Heft 2, S. 132138, 2005.

[10] Guandalini S., Burdet O. and Muttoni A.: Punching tests of slabs with low reinforcement ratios; ACI Structural Journal, Vol 106, No. 1, S 87-95, USA, 2009.

[11] Muttoni A., Schwartz J. und Thürlimann B.: Bemessung von Betontragwerken mit Spannungsfeldern, Birkhäuser Verlag, 143 S., 1997.

[12] DIN 1045-1:2008-08, Tragwerke aus Beton, Stahlbeton und Spannbeton, Deutsches Institut für Normung, Berlin, August, 2008.

[13] Nielsen M. P.: Limit analysis and concrete plasticity, 2nd Edition, CRC Press, 908 S, 1999.

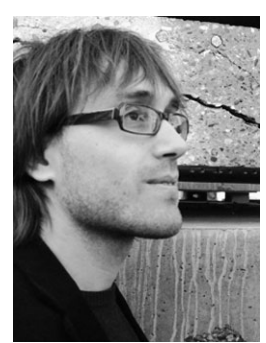

Dipl.-Ing. Roberto Guidotti roberto.guidotti@epfl.ch

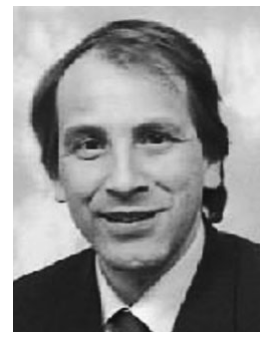

Prof. Dr.-Ing. Aurelio Muttoni aurelio.muttoni@epfl.ch

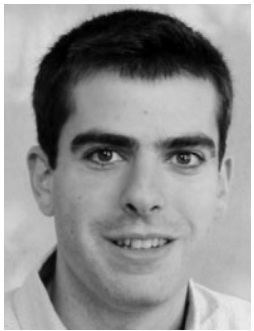

Dr.-Ing. Miguel Fernández Ruiz miguel.fernandezruiz@epfl.ch

Eidgenössische Technische Hochschule Lausanne Station 18 $\mathrm{CH}-1015$ Lausanne Schweiz

\section{Aktuelles}

\section{Betonieren von Wänden bei kühler Witterung}

In der kühleren Jahreszeit tritt beim Betonieren eine Verzögerung des Erstarrens und der Festigkeitsentwicklung ein. Gerade schlanke Bauteile, wie zum Beispiel Wände, müssen jetzt stärker vor dem Auskühlen geschützt werden.

Um den äußeren Einflüssen entgegen zu wirken, sollte darauf geachtet werden, dass bei Außentemperaturen $<5{ }^{\circ} \mathrm{C}$ ein Beton mit mindestens mittlerer Festigkeitsentwicklung verwendet wird. Entsprechende Rezepturen liegen auf jedem Transportbetonwerk bereit und können von den Kunden abgerufen werden. Diese Rezepturen sind mit schneller abbindenden Zementen versehen, die eine höhere Hydratationswärme entwickeln. Zudem sollten die Mindesttemperaturen für Frischbeton gemäß
DIN 1045-3 beachtet werden. Falls die Außentemperatur nur noch $0^{\circ} \mathrm{C}$ betragen sollte, sollte man den Einsatz von Warmbeton in Betracht ziehen. Bei Temperaturen $<-3^{\circ} \mathrm{C}$ sind Transportbetonhersteller verpflichtet, den Beton mit einer Temperatur von mindestens $10^{\circ} \mathrm{C}$ auszuliefern. Die geforderten Frischbetontemperaturen erreicht man mit warmem Anmachwasser oder Gesteinskörnung, die durch heißen Dampf aufgeheizt wurde.

Bereits im Vorfeld sollten mit dem Betontechnologen die Besonderheiten der Bauteile - wie z. B. Bauteilgeometrie und Standort - besprochen werden, um einen reibungslosen Ablauf des Betonierens zu gewährleisten. Vor dem Betonieren sollten die Schalung und die Bewehrung auf jeden Fall frei von Schnee und Eis sein. Die Baustelle muss den jungen Beton schützen, um eine ausreichende
Hydratation zu gewährleisten. Dies kann durch verschiedene Maßnahmen erreicht werden:

- Lange Standzeiten der Fahrmischer auf der Baustelle vermeiden, z. B. durch das Fördern über eine Betonpumpe.

- Schalung von außen zusätzlich mit Styropor dämmen, um die Randzone des Betons vor den kalten Temperaturen zu schützen.

- Jungen Beton mit wärmedämmender Abdeckung vor Frost und Austrocknen schützen, um eine ausreichende Hydratation zu gewährleisten.

- Auf eine Nachbehandlung mit Wasser verzichten.

- Beton so lange wie möglich in der Schalung belassen.

- Nachbehandlungsdauer nach DIN 1045-3, Abschnitt 8.7.4, unbedingt einhalten. 